\title{
Coordinated Exception Handling in J2EE Applications
}

\author{
Paweł L. Kaczmarek, Bogdan Krefft, and Henryk Krawczyk \\ Faculty of Electronics, Telecommunications and Informatics \\ Gdańsk University of Technology, Poland \\ pawel.kaczmarek@eti.pg.gda.pl, bogdan.krefft@bluemedia.pl, \\ henryk.krawczyk@eti.pg.gda.pl
}

\begin{abstract}
In the paper, we present a method of exception handling in $\mathrm{J} 2 \mathrm{EE}$ applications. It is proposed to create a dedicated component that is responsible for handling two types of exceptions: those concerning more than one object and those occurring commonly in an environment. The component, referred to as Remote Exception Handler, is an extension of the middleware layer of a computer system, which enables its use without modifications of application source code. Concerning highly distributed architectures, many cooperating Remote Exception Handlers placed on different nodes are created. The solution has been implemented in practice in JBoss Application Server as an additional service of the platform.
\end{abstract}

\section{Introduction}

Exception handling $(e h)$ is commonly used to increase software fault tolerance and consequently software dependability. An exception is a special event, usually a result of an error, that causes a change in the control flow and requires special handling. $E h$ in J2EE is based on the sequential Java language, although extensions have been designed. RemoteException is thrown if a remote object cannot fulfill a service or if there is a problem in communication with a server.

\subsection{Related Work on Exception Handling in Distributed Environments}

Distributed programming platforms supply a range of extensions that support fault tolerance and application management. The J2EE environment is equipped with Java Management Extension (JMX) 1] as a means of management and monitoring of applications, devices, services and JVM. Container Managed EH [2] (CMEH) is proposed as a server-side fault tolerance mechanism in J2EE application servers. CMEH framework is based on intercepting calls of component methods by an interceptor augmented with $e h$ features. A system of recovering from exceptional situations by microreboots has been proposed in the JAGR system [3]. The $\mathrm{H} 2 \mathrm{O}$ system creates a reliable platform of distributed computing, including self-organizing applications [4. Eh in distributed environments is also addressed in [5] [6] and [7] for different platforms. 
Our solution differs from the ones described above as we propose specialized components that can cooperatively handle exceptions. We focus on the J2EE environment. We present the theoretical concept and describe the implementation of our solution in following sections. The source code of the implementation is available at author's web page (see Sect. 3).

\section{Local and Remote Exception Handling}

$E h$ in distributed systems may require the knowledge about system state or cooperation between a group of objects. Additionally, there are exceptions that occur commonly in an environment.

We propose a dedicated Remote Exception Handler $(R E H)$ that supplies predefined handling functions. $R E H$ extends the middleware layer by adding a service for handling two kinds of exceptions: those occurring commonly in the environment and those concerning more than a single object. $R E H$ operates in three main fields: performing handling actions, suggesting repair actions to objects and gathering information about system state.

Application programmer is freed from implementing handling functions, which reduces the required work and enables faster application development. As an example consider that a group of objects throw repeatedly runtime exceptions (e.g. a corrupted data base access pol). $R E H$ detects the situation and eliminates the cause (creates a new pol). The automated repair applies to those exceptions that are not part of the business specification (e.g. an input value exceeds a bound).

\subsection{Integration of $R E H$ with a Distributed System}

$R E H$ is integrated with an existing distributed system in three alternative ways:

- with interceptor cooperation - an exception is caught by an interceptor and redirected to $R E H, R E H$ performs a handling function, it returns a normal result or throws an exception if the handling fails,

- direct cooperation with an object or service - if an object detects an anomaly, it invokes an $R E H$ method to repair the situation,

- self-activation - REH performs independently a handling function.

The methods of integration require some modifications in the middleware layer as explicit invocations of $R E H$ methods need to be added. It is also possible to extend $R E H$ 's functionality with application specific handling functions. A detailed description of $R E H$ implementation and use is presented in Sect. 3 .

\subsection{Multi-remote Exception Handler Architecture}

Many $R E H$ components are proposed for highly distributed architectures to overcome reliability and performance problems. The multi- $R E H$ architecture, however, requires additional solutions to enable inter- $R E H$ communication and synchronization. On $R E H$ creation, the component sends notification to other 
$R E H$ s to register its existence. Each component maintains a list of active $R E H \mathrm{~s}$ together with their references. They communicate with each other during execution to interchange information about system and application state. It is assumed that inter- $R E H$ communication is reliable for active (visible) $R E H$ components. Only those components participate in a recovery. Fig. 1 shows the architecture of multi- $R E H$ cooperation.

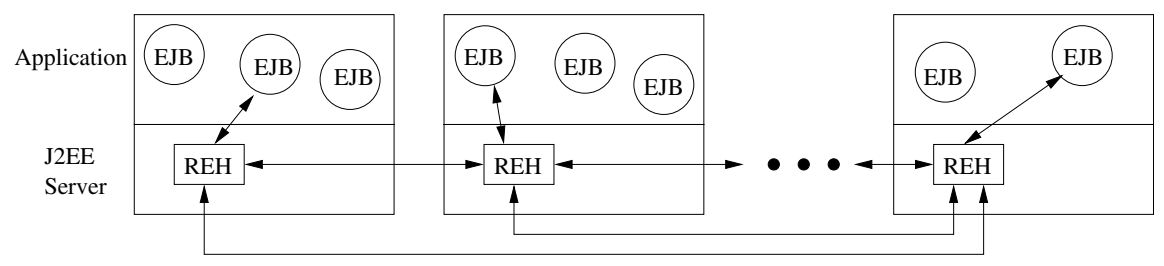

Fig. 1. Multi- $R E H$ architecture

Typically, $R E H$ performs repair actions independently, however, it can communicate with another $R E H$ to request the handling of an exception. The second handler attempts the handling and returns the result. In the current implementation, each $R E H$ sends a request to another arbitrary $R E H$. If the request fails in the second handler, an exception is returned to the caller.

\section{Exception Controllers in JBoss Application Server}

The $R E H$ architecture has been implemented in JBoss Application Server [8]. The JBoss architecture consists of a micro-kernel and components implementing individual services. JBoss is an open-source server that can be easily extended.

The REH implementation in JBoss consists of two subcomponents as new JBoss services: Local Exception Controller $(L E C)$ and Remote Exception Controller $(R E C)$. $L E C$ implements basic exception handling scenarios that concern a single instance of the server. $R E C$ implements inter- $R E H$ cooperation, registration and communication between different instances of JBoss servers.

The creation and maintenance of $R E H$ s requires similar resources to other services in JBoss. Sending a request to a $R E H$ component is comparable to a remote invocation. Therefore, the performance degradation of a system using $R E H$ depends on the number of thrown exceptions.

The source code of the implementation and its configuration files are available at author's web page. The page contains also an exemplary application:

www.eti.pg.gda.pl/ pkacz/j2ee_exception.html

\subsection{Required Configuration}

The implemented extensions need to be integrated with JBoss, which requires configuration changes. Most changes concern the JBoss environment, however, minor modifications must be made in application deployment descriptors. The 
following configuration items are involved: deployment descriptors of $L E C$ and $R E C$, the configuration of remote servers cooperating with the local $R E C$, the configuration of the EJB container. The first two points concern configuration changes made in JBoss independently of any application. The last point concerns configuration changes required in an application run on the platform: bean's container must be specified and container definition must be copied.

\section{The Use of Exception Controllers}

The implemented extension has been tested on an exemplary application - Duke's Bank. Supposing an EJB method throws a non-business exception (see Sect. 22), examples of handling functions are: (i) simple reinvocation - the interceptor catches the exception and requests $L E C$ to reinvoke the method, (ii) ordering a reinvocation on a remote server, (iii) running garbage collection - if the exception is OutOfMemory, $L E C$ enforces garbage collection to free unused memory, the request is repeated, (iv) invalidating a bean - it is assumed that bean's invocations can degrade the system, further invocations are rejected.

An explicit invocation of a $L E C$ method from a regular object is also possible, which requires the same operations as a typical remote invocation: looking up the JNDI and creating object's reference.

Exception handling in distributed systems can be supported with the use of Remote Exception Handler, which extends the range of handled exceptions. After the extensions are made to the middleware layer of a system, $R E H$ can be used by all applications running in the environment, which seems a significant advantage of the solution. The work was supported in part by KBN under the grant number $4 \mathrm{~T} 11 \mathrm{C} 00525$.

\section{References}

1. Armstrong, E., Ball, J., Bodoff, S.: The J2EE 1.4 Tutorial. Sun Microsystems Inc. (2004)

2. Simons, K., Stafford, J.: Container-Managed Exception Handling Framework. Department of CS, Tufts University, Medford, MA, USA (2004)

3. Candea, G., Kiciman, E., Zhang, S., Keyani, P.: JAGR: An Autonomous SelfRecovering Application Server. In: 5th Int. Wrkshp on Active Middleware Services. (2003)

4. Kurzyniec, D., Wrzosek, T., Drzewiecki, D., Sunderam, V.: Towards Self-Organizing Distributed Computing Frameworks: The H2O Approach. Parallel Processing Letters (2003)

5. Feitelson, D.G.: Exception Propagation in the ParPar System. Technical report, Inst. of Comp. Science, The Hebrew Univ. of Jerusalem (1998)

6. Romanovsky, A.: Practical Exception Handling and Resolution in Concurrent Programs. Comput. Lang. Vol. 23 (1997)

7. Kaczmarek, P.L., Krawczyk, H.: Remote Exception Handling for PVM Processes. LNCS 2840 Conf. X Conf. EuroPVM/MPI (2003)

8. JBoss Inc.: JBoss Admin Development Guide. (2004) 